\title{
$N$-Acetylglucosamine-6-Sulfate Sulfatase in Man: Deficiency of the Enzyme in a New Mucopolysaccharidosis
}

\author{
LEONARD C. GINSBERG, PATRICIA V. DONNELLY, DANIELA T. DI FERRANTE, \\ NICOLA DI FERRANTE, ${ }^{(53)}$ AND C. THOMAS CASKEY \\ Laboratories of Connective Tissue Research of the Department of Biochemistry (L.C.G., P.V.D., D.T.DiF., N.DiF.) \\ and the Division of Medical Genetics of the Department of Medicine (C.T.C.), Baylor College of Medicine,
}

Houston, Texas, USA

\section{Summary}

The study of a 5-year old patient with a mucopolysaccharidosis different from those already known has given the opportunity to describe the clinical, biochemical and enzymic characteristics of $\mathrm{N}$-acetylglucosamine-6-sulfate sulfatase deficiency.

The patient was delayed physically and mentally, was hyperactive, and had a short attention span. He had fair complexion and very blond, but coarse and excessive hair. He had mild hepatosplenomegaly and dysostosis multiplex, including hypoplastic odontoid process of the atlas, ovoid vertebral bodies, small femoral epiphyses, and modest enlargement of the ribs. He had a mild, bilateral conductive hearing loss. The urine gave a strongly positive spot test with Azur A paper. Quantitative measurement of urinary glycosaminoglycans demonstrated increased excretion and inadequate degradation of heparan sulfate and keratan sulfate. The microscopic examination of the stained peripheral leukocytes revealed metachromatic material which formed a ring on the inner aspect of the cellular membrane in $20 \%$ of the lymphocytes. The rate of degradation of ${ }^{35} \mathrm{SO}_{4}$-labeled intracellular glycosaminoglycans by cutaneous fibroblasts was delayed and inadequate.

The measurements of several lysosomal enzymes whose deficiencies are responsible for the known mucopolysaccharidoses gave results within normal ranges. Galactose-6-sulfate (Gal-6-S), $\boldsymbol{N}$-acetylgalactosamine-6-sulfate (GalNAc-6-S), and $\mathbf{N}$-acetylglucosamine-6-sulfate (GlcNAc-6-S) were prepared with chlorosulfonic acid, according to the method of Suzuki and Strominger (38). After chromatographic purification, aliquots of the three 6sulfated monosaccharides were reduced with sodium borotritide. Measurements of Gal-6-S sulfatase, GalNAc-6-S sulfatase, and GlcNAc-6-S sulfatase were performed on extracts of normal cul- tured fibroblasts and of fibroblasts of the propositus, his parents, and several Morquio patients. Additionally, GalNAc-6-S sulfatase and GlcNAc-6-S sulfatase activities were also measured, using as substrate $500 \mathrm{nmol}$ tetrasaccharide obtained from either chondroitin-6-sulfate or nonradioactive GlcNAc-6-S. The measurement demonstrated that the propositus has a defective GlcNAc-6-S sulfatase activity, but normal activities for Gal-6-S and GalNAc6-S sulfatase.

These findings are in contrast with those found with fibroblast extracts of Morquio patients, since they have low or nondetectable activities of Gal-6-S and GalNAc-6-S sulfatase but normal GIcNAc-6-S sulfatase activity.

Extracts of leukocytes and fibroblasts of the propositus' parents, when tested with radioactive or nonradioactive substrates, had normal levels of GalNAc-6-S sulfatase activity but decreased levels of GlcNAc-6-S sulfatase activity (50-60\% of normal).

\section{Speculation}

The possible existence of two different sulfatases, specific for 6 sulfated hexoses with the glucose or galactose configuration, has been suggested (10).

The study of a patient with a novel mucopolysaccharidosis, characterized by defective degradation of keratan sulfate and heparan sulfate, has provided the opportunity of describing the clinical and biochemical features of GIcNAc-6-S sulfatase deficiency. These findings confirm that the defective degradation of keratan sulfate and chondroitin-6-sulfate typical of classic Morquio disease is caused by the deficiency of a sulfatase specific for Gal-6-S and GalNAc-6-S. 
The pathogenesis of several mucopolysaccharidoses has been clarified during the last ten years essentially by the experimental work performed in the laboratorles of Dorfman and Neufeld. Using cultured skin fibroblasts, Neufeld and collaborators $(12,13)$ demonstrated that those derived from affected patients had a defective degradation of intracellular glycosaminoglycans (GAG) which could be corrected by the addition to their cultures of concentrates of mediur of normal fibroblasts or of fibroblasts derived from patients with a different mucopolysaccharidoses. These findings led Dorfman and collaborators (11) to the use of natural or synthetic substrates for the identification of enzymes involved in the degradation of the stored GAG. Thus, following these different and complementary approaches, the enzyme deficiencies responsible for the impaired degradation of dermatan sulfate and/or heparan sulfate (DS, HS) which occurs in Hurler/Scheie $(3,27,40)$, Hunter $(2,36)$, Sanfilippo A $(20)$ and $\beta-$ glucuronfdase deficiency (17) diseases were 1dentified. Subsequently, the
enzyme deficlencles occurring in Sanfilippo B (29) and Maroteaux-Lamy (37) diseases have also been identified.

In contrast with the rather close sequence in which those discoveries were made, the identification of the enzyme deficiencies related to impaired degradation of keratan sulfate (KS) has been delayed and made wore difficult by the impossibility of studying KS metabolis
in fact, do not synthesize KS.

Morquio disease became separated from the various osteochondrodysplasias when it was demonstrated that affected patients have excessive keratansulfaturia (30) and accumulate KS and chondroitin-6-sulfate (C-6-S) in cartilage (31). In 1974 Matalon et al. (26) performed enzyme measurements with biologically labeled, polymeric $35 \mathrm{SO}_{4}$-chondroitin-4/6-sulfate and ollgosaccharides derived from 1t and concluded that a hexosamine-6-sulfate sulfatase activity was absen or greatly decreased in homogenates of Morquio skin fibroblasts. As a result of these findings it vas suggested that the impaired degradation of KS and C6-S in Morquio disease could be ascribed to the deficiency of an enzyme normally active on N-acetylglucosamine-6-sulfate (GleNAc-6-S) and galactose-6sulfate (Gal-6-S) residues of KS and on $\mathrm{N-acetylgalactosamine-6-sulfate} \mathrm{(Gal}$
NAc-6-S) residues of C-6-S (10). In 1976 Singh et al. (33) used as substrate NAc-6-S) residues of C-6-S (10). In 1976 Singh et al. (33) used as substrate
tetrasaccharides prepared from C-6-S and suggested that the enzyme defective in tetrasaccharides prepared from $\mathrm{C}-6-\mathrm{S}$ and
Morquio disease was GalNAc-6-S sulfatase.

Because an impaired degradation of HS (which contains N-acetyl and N-sulfated glucosamine-6-sulfate residues) has not been found to be present in Morquio disease, Neufeld suggested that different 6-sulfatases might exist, specific either for the glucose or the galactose configuration of their substrates
(quoted in (10)). If this hypothesis were correct, deficiency of a sulfat (quoted in (10)). If this hypothesis were correct, deficiency of a sulfatase normally active on Gal-6-S and on GalNAc-6-S should be associated with Morquio hitherto unknown syndrome of Impaired degradation of KS and HS.

The study of a five year old patient with a mucopolysaccharidosis different from those already known has given us the opportunity to demonstrate the correctness of Neufeld's hypothesis.

In previous publications we have provided evidence that classical Morquio disease is associated with defective Gal-6-S and GalNAc-6-S sulfatase activity the patient affected by GlcNAc-6-5 sulfatase deficlency.

\section{Clinical description} G.G., a five year old Caucasian male, only live of fspring of a nonconsanguin-
eous couple, was the product of term pregnancy and normal delivery. At birth he weighed $3.5 \mathrm{Kg}$ and had fronto-occipital circumference and body length of 31.5 and $49.5 \mathrm{~cm}$, respectively. Thirteen hours after birth he developed cyanos1s, thought to be caused by hyaline membrane disease, and
he was kept one week in atmosphere of oxygen supplementation.

At eight months of age he could stand and use three words; at 11 months he could walk and at three years he could use short phrases, ride a tricycle and dress himself.

At age 14 months he had febrile selzures characterized by extension of the extremities. These eplsodes occurred again twice, and for the last time, at two and one-half years of age. Since then, the patient has been maintained on phenytoin sodium (Dilantin).

Because of his delayed physical and mental development, the patient has been evaluated repeatedly. When three years, three months of age he was found to have the mental language of a 21 month old child. At four years of age, he was found to be able to use several sentences, to be hyperactive and to have a
short attention span. S1x months later, a Peabody Picture Vocabulary test gave a score of 59 and a Slosson Intelligence test of 69 . S1x months later, these a score of 59 and a Slosson Intelligence test of 69 . Six months later, these
tests gave scores of 62 and 58 , respectively, and an I.Q. of 50-60. He has tests gave scores of 62 and 58 , respectively, and an I.Q. of $50-60$.
been attending special education classes, up to the present time.

The family history does not reveal children simflarly affected. The parents have had two additional pregnancies, one terminated spontaneously at the first
trimester, the other terminated electively.

Physical examination reveals a hyperactive male, $100 \mathrm{~cm}$ high (less than third percentile), weighing $18.6 \mathrm{Kg}$, with fair complexion and capable of following instructions. The head and chest circumferences are 54 and $59.9 \mathrm{~cm}$, respectively. The facies is not remarkable, but the ha1r, very blond, is coarse and excessive. There is no evidence of corneal clouding, gum hyperplasia, lymphoadenopathy or thyroid enlargement, cardiomegaly or cardiac murn

The skeletal radiographic survey shows features of dysostosis multiplex, to include J-shaped sella turcica; hypoplastic and dysplastic odontoid process of the atlas; ovoid shape of the lower dorsal vertebral bodies, with bulging of the vertebral inplates; small femoral epiphyses with horizontal acetabular roofs; modest enlargement of the lateral portion of the ribs and of the medial and of the distal ends of the proximal phalanges.

Audiometric examination reveals mild, bilateral conductive hearing loss.

Routine laboratory examinations were normal and did not reveal any impairment of liver function or of the blood clotting system. Hematologic examination was normal, except for the presence of metachromatic granulations in peripheral leukocytes (see Methods and Results).
METHODS

The urinary excretion of polymeric GAG and of oligosaccharides was measured on two sporadic urine specimens collected in January and April 1976 and on a 24 hour specimen collected in October 1976, using previously described techniques $(7,9,23)$. Additional procedures, modified from those of Wolfe et al. (41) wer
used to investigate whether some urinary GAG did not precipitate with cetylused to investigate whether some urinary GAG did not precipitat
pyridinium chloride (CPC) (42) and remained in the supernatant.

The presence of Reilly bodies in peripheral leukocytes was investigated as follows: $10 \mathrm{ml}$ of venous blood were mixed in a vertical test tube with $4 \mathrm{ml}$ of polyvinylpyrrolidone solution (PVP-40, Sigma Chemical Co. (43), M. W. 40,000; $3.5 \mathrm{~g} / 100 \mathrm{ml}$ saline) and $0.1 \mathrm{ml}$ of aqueous $0.27 \mathrm{M}$ ethylenediaminotetracetate
solution (2.05 g EDTA and $7.98 \mathrm{~g}$ of EDTA, tetrasodium salt/liter). Two hours later the supernatant was removed by aspiration and centrifuged at room temperature for 10 minutes at $700 \mathrm{~g}$. The supernatant was discarded and aliquots of ature for 10 minutes at $700 \mathrm{~g}$. The supernatant was discarded and aliquots of were air dried, flxed in absolute mether formal blood smears. The slides minutes in $0.5 \%$ solution of tolufdine blue (44) in $25 \%$ acetone in water. The slides were washed in three changes of absolute acetone for $10-15$ seconds each, slides were washed in three changes of absolute acetone for $10-15$ seconds eac
cleared for 1 minute in xylene and examined for intracellular metachromatic material with the microscope, under oil immersion.

Normal and mutant cultured skin fibroblasts (informed consent was obtained before performing skin biopsies), cultured as described previously (34) were removed by trypsinization from one or more $75 \mathrm{~cm}^{2}$ plastic plates and collected by centrifugation (34). The pellets, suspended $1 \mathrm{n} \mathrm{I} \mathrm{ml}$ of saline and immersed in an 1ce bath, were sonicated for 20 seconds (Heat Systems (45) sonicator model W200R, 30 watts, microtip). The homogenates were dialyzed overnight at $4^{\circ} \mathrm{C}$ against saline and the retentates were cleared by. centrifugation at 20,000 $\mathrm{B}$ for 10 minutes at $4^{\circ} \mathrm{C}$. Allquots of the clear supernatants were used for
protein measurement $(25)$ and enzyme assays. Additional plates of cultured skin fibroblasts from the propositus and a normal control were used as previously fibroblasts from the propositus and a normal control were used as previously
described (34) for the measurements of $35 \mathrm{SO}_{4}$ uptake and degradation of $35 \mathrm{SOL}_{-}$ described (34) for the measurements of $3 \mathrm{SO}_{4}$ uptake and degradation of $35 \mathrm{SO}_{4}-$ concentrate of medium obtained from Sanfilippo A cultured fibroblasts was added to cultures of the propositus' fibroblasts.

Gal-6-S, GalNAc-6-S and GIcNAc-6-S were prepared with chlorosulfonic acid, according to the method of Suzuki and Strominger (38), using D-galactose (F1sher Scientific Co. (44)), N-acetyl-D-galactosamine (Sigma Chemical Co.
(43), Lot 26C-0103-1) and N-acetyl-D-glucosamine (Chas. Pfizer \& Co. (46), Lot 86146-05-EPD) as starting materials. The barium salts of the sulfated products, separated from the respective non-reacted material by precipitation with absolute ethanol, were first converted to the potassium salt by chromatography through Dowex $50 \times 8(48)$ in the $\mathrm{H}^{+}$form, followed by neutralization, with a 18 KOH solution, and then desalted by gel filtration on Biogel P-2 (47) columns
$(2 \times 180 \mathrm{~cm}$, 50-100 mesh) packed and eluted with water. The products were (2 $\times 180 \mathrm{~cm}, 50-100$ mesh) packed and eluted with water. The products were purified by paper chromatography, using solvent $B$ (butanol, glacial acetic
acid, I $\mathrm{N} \mathrm{NH} 4 \mathrm{OH} 2: 3: 1$ ) of Dletrich, Silva and Michelacc1 (6). The various

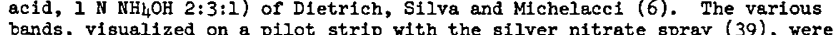
bands, visualized on a pilot strip with the silver nitrate spray (39), were eluted with water, lyophilized and analyzed by infrared spectroscopy; for sul-
fate content (16), after hydrolysis with $25 \%$ formic acid for 14 hours at $110^{\circ} \mathrm{C}$ for neutral sugars (32), reducing groups (21) and hexosamine content (14) in order to determine the sulfate/hexose molar ratios.

Subsequently, preparations of the three 6-sulfated monosaccharides were reduced with sodium borotritide according to Horton and Philips (19) and the specific activity of each [1-3H]-labeled hexitol-6-sulfate was calculated as cpm/nmole

\section{Enzyme studies}

Measurements of $\alpha-L$-iduronidase (28), L-idurono-2-sulfate sulfatase (15), $\alpha-N-$ acetylglucosaminidase (34), B-galactosidase (5), N-acetylgalactosamine-4-
sulfate sulfatase (arylsulfatase B) (35) and B-glucuronidase (34) were performed using as a source of enzyme either the propositus' serum or leukocytes extracts.

Measurements of Gal-6-S sulfatase, GalNAc-6-S sulfatase and GlcNAc-6-S sulfatase vere performed on extracts of normal cultured fibroblasts and of fibroblasts derived from the propositus, his parents and several Morquio patients
with the following method. Fifty $\mu 1$ of fibroblasts extracts in saline and with the following method. Fifty $\mu 1$ of fibroblasts extracts in saline and
$10 \mu 1$ of $0.6 \mathrm{M}$ acetate buffer $\mathrm{pH} 4.8$ (containing $5 \%$ bovine serum albumin, Miles (48)) were mixed in plastic centrifuge microtubes with $40 \mu 1$ of water containing efther 100 nmol of [ [1-3H]-galactitol-6-sulfate (2700 cpm/nmole) or 150 nmol of [ $1-3 \mathrm{H}]-\mathrm{N}-\mathrm{acetylgalactosaminitol-6-sulfate}(1200 \mathrm{cpm} / \mathrm{nmole})$ or $180 \mathrm{nmol}$
of $[1-3 \mathrm{H}]-\mathrm{N}-\mathrm{ac}$ etylglucosaminitol-6-sulfate $(1200 \mathrm{cpm} / \mathrm{nmole})$. Control tubes, containing only the fibroblasts extract in buffer or each of the substrates in water, were also prepared. All these tubes were incubated at $37^{\circ} \mathrm{C}$ for 5 hours. Upon termination of the 1ncubations the enzyme and substrates control tube were mixed and all the tubes were 1mmerged for 2 minutes in boiling water. After centrifugation for 1 minute at $10,000 \mathrm{~g}$ in a microfuge, each clear supernatant was aspirated, added to $0.8 \mathrm{mI}$ of water and applied to a column of Dowex $1 \times 8$ (47) ( $5 \times 20 \mathrm{~mm}, 200-400$ mesh, $\mathrm{Cl}^{-}$form), packed in water. Because of their anionic charge, the sulfated substrates are retained on the column, while the desulfated products are not. Each water effluent, plus $0.3 \mathrm{ml}$ of water
wash, were collected directly into counting vials, containing $10 \mathrm{ml}$ of Aquasol wash, were collected directly 1nto counting vials, containing $10 \mathrm{ml}$ of Aquasol
(New England Nuclear (49)), cooled and counted for 20 minutes in a Packard TriCarb spectrometer. Results were expressed as nmoles of product obtained in on hour/mg protein. These were calculated by subtracting the cpm of the control tubes ( $1 \%$ of the total cpm incubated in the case of galactosaminitol-6-sulfate and glucosaminitol-6-sulfate; 4\% in the case of galactitol-6-sulfate) from the $\mathrm{cpm}$ of the corresponding tubes in which the substrate was incubated with fibro blasts extracts. Fibroblasts extracts with normal enzyme activities had a minimum of $3000 \mathrm{cpm}$ above control values while those
varied between 0 and $100 \mathrm{cpm}$ above control values.

Additionally, GalNAc-6-S sulfatase activity of homogenates of normal fibroblasts, of Morquio fibroblasts and of the propositus' fibroblasts was measured with a micro-modification of the method described by Singh et al. (33), using as a substrate $500 \mathrm{nmol}$ of tetrasaccharides obtained from C-6-S. GlCNAc-6-S. sulfatase activity of the same homogenates was also measured as described
using $500 \mathrm{nmol}$ of non-radioactive, synthetic GlcNAc-6-S and an incubation period of 24 hours. In both cases, enzymic activity was expressed in terms of inorganic sulfate released in one hour per mg protein, the sulfate being measured with a modification of the rhodizonate method (16).

RESULTS

The propositus' urine always gave a strongly positive "spot test" with Azur A paper (49). Quantitative measurements of the urinary GAG (Table I) demon-
strated an increased excretion and inadequate degradation of hexuronate-con- 
taining GAG as indicated by the high rat10 polymeric GAG/oligosaccharides. HS the total. The latter GAG also appeared to be Inadequately degraded.

Since it is known that KS (galactose-containing GAG) is not completely precipitated with CPC (1), it was measured not only in the CPC precipitate but also in the CPC supernatant. The latter was digested with pronase $(50)$ and dialyzed; the non-dialyzable material was precipitated with absolute ethanol. The large distributed between CPC precipitate and supernatant.

The microscopic examination of the stained peripheral leukocytes revealed that in $20 \%$ of the lymphocytes, the 1ntracellular metachromatic material formed a ring on the inner aspect of the cellular membrane, while in 1-2\% of the granu-

Figure 1 shows that appreciable differences were not found when the rate of $35 \mathrm{SO}_{4}$ uptake by the propositus' fibroblasts was compared to that of control fibroblasts. Vice versa, the rate of degradation of $35 \mathrm{SO}_{4}$-labeled, intracellular GAG appeared to be delayed and inadequate throughout the period studied. This Inadequate degradation was corrected when a concentrate of medium derived from Sanfilippo A cultured fibroblasts was added to the propositus' culture at the beginning of the experiment.

The Infrared analyses of the purlfled 6-sulfated monosaccharldes prepared for the measurement of the various 6-sulfatases showed a strong absorption band at $1235 \mathrm{~cm}^{-1}$, typical or $\mathrm{S}-0$ stretching, and minor ones at 775,825 and $995-1000$ cm-1, Indicative of sulfate groups in the 6 position. No spectroscopic evicharlde and sulfate content of the three preparations gave the following Bulfate/hexose molar ret

The measurement of several lysosomal enzymes whose deficlencles are responsible for the known mucopolysaccharidoses, when performed on the propositus' serum or on extracts of his leukocytes gave results well within the normal range (Table II). However, the measurement of the varlous 6-sulfatase activities performed with the various gubstrates prepared, elther radioactive or non radioactive, demonstrated (Table III) that the propositus has a defective $6 \rightarrow 5$ sulfatase.

These findings are in contrast with those found with fibroblasts extracts of and GalNAc-6-S sulfatase but normal GlcNAc-6-S sulfatase activity.

Data not presented have demonstrated that normal enzymic activity was not inhlblted when extracts of normal fibroblasts were mixed, in several proportions, with extracts of the propositus' fibroblasts.

Extracts of leukocytes and fibroblasts of the propositus' parents, when tested with radioactive or non-radioactive substrates (Table III) had normal GalNAc-

\section{DISCUSSION}

The conspicuous excretion of urinary GAG, with positive Azur A spot test, and the presence of metachromatic inclusions in the peripheral leukocytes indicate that the propositus is affected by a mucopolysaccharidosis.

Qualitative analyses of the urinary GAG have demonstrated that they cons1st essentialiy of polymeric KS and HS. Since keratansulfaturia does not give a positive Azur A test, this must be attributed to the 1ncreased excretion of HS
and other hexuronate-containing GAG. The excretion of HS 1s certalnly larger and other hexuronate-containing GAG. The excretion of HS is certainly larger than that reported in Table I in terms of mg of 2-deoxy-2-sulfoeminohex (22), because approximately $50 \%$ of the glucosamine present 1 in HS is $\mathrm{N}-$
acetylated $(4,24)$ and unreact1ve with the nitrous ac1d-indole reaction of Lagunoff and Warren $(22,23)$.

Since this type of mucopolysaccharldurla and the particular distribution of intracellular metachromatic material have not been reported previously, it seemed possible that the patient could be affected by a new mucopolysaccharidosis, possibly of the type suggested by Neufeld to be caused by the deficiency of an enzyme normally particlpating in the degradation of KS and HS.

This possibility was supported by the clinical and radiological examinations of the patient (Table IV) which demonstrated a phenotype characterized by the association of findings typical of diseases in which HS or KS accumulate (28). In fact, the mental retardation, hyperactivity, excessive and coarse hair and history of selzures, typical of Sanfillppo diseases, were found to be associated with retarded growth, dysplasis of the odontold process of the atlas and

On the other band, it was clear that the patient did not have any of the known mucopolysaccharioses. In fact, the measurements of the various lysosomal enzymes listed in Table II eliminated the diagnosis of Hurler/Scheie, Hunter, Sanf1lippo B, Maroteaux-Lamy and B-glucuronfdase deficiency. That of Morquio disease was very 11 kely excluded because of the excessive heparansulfaturia an the delayed degradation of intracellular GAG by the patient's cultured fibroblasts. Finally, the correction of this parameter 1n presence of media con centrate from Sanfilippo A fibroblasts
excluded the diagnosis of Sanfilippo A.

To demonstrate that the patient was affected by a mucopolysaccharidosis with Impaired degradation of KS and HS, it became necessary to prepare a substrate for the measurement of a sulfatase active on the sulfate present in position of GlcNAc residues, since this 1s the only functional group common to the repeating unit of KS and HS (F1g. 2). And slnce the postulation of the existence of this sulfatase requires the assumption that at least another one must exist, active on the sulfate present in position 6 of Gal or GalNAc, we prepared several radioactive and non-radioactive substrates which would be tose configuration of their substrates.

We have already reported that extracts of cultured skin fibroblasts of Morquio patients, when incubated with tetrasaccharides prepared from C-6-S, fail to release measurable amounts of inorganic sulfate ( 8 ). We have also demonstrated that those extracts fa1] to desulfate either [1-3H]-galactitol-6-sulfate or [1-3H]-N-acetylgalactosaminitol-6-sulfate, while they desulfate normally el ther radioactive [1-3H]-N-acetylglucosaminitol-6-sulfate or non-radioactive GlcNAc$6-5$. As a result of these findings, it is clear that the impaired degradation and accumulation of $\mathrm{KS}$ and $\mathrm{C}-6-\mathrm{S}$ in Morquio disease is caused by the deficiency of a sulfatase specific for the Gal-6-S residues of $\mathrm{KS}$ and the GalNAc-6-S resi-
dues of $\mathrm{C}-6-\mathrm{S}$ (Fig. 2). V1ce versa, extracts of cultured skin fibroblasts of our patient desulfate normally elther tetrasaccharides from C-6-S or radioactive $[1-3 \mathrm{H}]-$ galactitol-6-sulfate or [1-3H]-N-acetylgalactosaminitol-6sulfate. However, the same extracts do not desulfate GlcNAc-6-S or have minimal activity when incubated with radioactive [1-3H]-N-acetylglucosaminitol-6sulfate.

The higher activities obtained with the non-radioactive substrates in the experiments surmarized in Table III might be elther due to the higher concentrations of substrates used (about twice the $\mathrm{K}_{\mathrm{m}}$ values found for the crude enzymes) or to higher enzyme affinities for substrates not reduced at position 1 .

The enzymic activities measured when the varlous substrates vere incubated with extracts of flbroblasts or leukocytes derived from the propositus' parents demonstrate their heterozyous state and the autosomal recessive character of the genet1c defect.

In view of the feasibility of preparing adequate amounts of radioactive substrates of high specific activity, it is reasonable to presume that detailed knowledge of the various kinetic parameters of the enzyme GlcNAc-6-S sulfatas of the new disease.

\section{CONCLUSION}

A five year old male had retarded growth (less than third percentile), mental retardation (I.Q. 50-60), hyperact1vity, m1ld osteochondrodystrophy, hepatosplenomegaly, excessive and coarse halr, clear corneas and mild deafness. Excessive mucopolysacchariduria, consisting of keratan and heparan sulfate, and unusual metachromasia of the peripheral lymphocytes were present. The release of $35 \mathrm{SO}_{4}$ incorporated by h1s cultured akin fibroblasts was delayed. All the known mucopolysaccharidoses were excluded on the basis of spec1f1 1 enzyme measurements and of experiments in which the delayed release of $35 \mathrm{SO}_{4}$-labele glycosaminoglycans by his fibroblasts was corrected with extracts of fibroblast derived from patients affected by known mucopolysaccharidoses. Since both keratan and heparan sulfate have N-acetylglucosamine-6-sulfate residues, this substrate was synthesized as described by Suzuk1 and Strominger ( 38 ). The product, which had proper analysis and infrared spectrum, was desulfated by extracts of normal fibroblasts and flbroblasts derived from Morquio patient but not by extracts of the propositus' flbroblasts. Simflar results were obtained using as a substrate [1-3H]-8lucoseminitol-6-sulfate, obtalned by reduct1on w1th sodium borotritide. Vice versa, extracts of the propositus f blasts desulfated-6ormale and 6-sulfated tetrasacharides derived from chondroltin-6-sulfate, while those of Morquio patients did not.

It 1 s concluded that Morquio disease and the mucopolysaccharldosis described are caused respectively by the deficlency of sulfatases specific for the galactose or glucose configuration of thelr 6-sulfated substrates.

\section{REFERENCES AND NOTES}

1. Antonopoulos, C.A., Borellus, E., Gardell, S., Hamnstrom, B., and Scott, J.E.: The precipltation of polyanions by long-chain allphatic ammonium compounds. Bloch1m. B1ophys. Acta, 54: 213 (1961).

2. Bach, G., Elsenberg, F., Jr., Cantz, M., and Neufeld, E.F.: The defect Natl. Acad. Sc1. USA, 70: 2134 (1973).

3. Bach, G., Frledman, R., Weismann, B., and Veufeld, E.F.: The defect in the Hurler and Schele syndromes: depis
Nat1. Acad. Sc1. USA, 69: 2048 (1972).

4. Cifonelli, J.A.: Reaction of heparitin sulfate with nitrous acid. Carbohyd. Res., 8: 233 (1968).

5. Conchie, J., and Hay, A.J.: Mammalian giycosidases. Blochem. J., 73: 327 (1959).

6. Dletrich, C.P., Silva, M.E., and Michelacc1, Y.M.: Sequential degradation of

7. Di Ferrante, N., Donnelly, P.V., and Berglund, R.K.: Colorimetric

8. Di Ferrante, N., Ginsberg, L.C., Donnelly, P.V., Di Ferrante, D.T., and Caskey, C.T.: Deficiencies of glucosamine-6-sulfate or galactosamine-6sulfate sulfatase are responsible for different mucopolysaccharidoses. Science, 199: 79 (1978).

9. D1 Ferrante, N., Ner1, G., Ner1, M.E., and Hogsett, W.E.: Mesurement of urinary glycosaminoglycans with quaternary ammo
of the method. Conn. T1ss. Res., 1: 93 (1972).

10. Dorfman, A., Arbogast, B., and Matalon, R.: The enzymle defect in Morquio and Maroteaux-Lamy syndrome. Adv. Exp. Med. Biol., 68: 261 (1976).

11. Dorfman, A., and Matalon, R.: The mucopolysaccharidoses. Proc. Natl. Acad. Sc1. USA, 73: 630 (1976).

12. Fratanton1, J.C., Hall, C.W., and Neufeld, E.F.: The defect In Hurler's and Hunter's syndrones: faulty degrada

13. Fratantoni, J.C., Hall, C.W., and Neufeld, E.F.: The defect In Hurler and Hunter syndromes. II. Deficlency of specific factors involved in mucopol.

14. Gatt, R., and Berman, E.R.: A rapid procedure for the est1mation of amino sugars on a micro scale. Anal. Biochem., 15: 167 (1966).

15. Ginsberg, L.C., Di Ferrante, D.T., and Di Ferrante, N.: A substrate for direct measurement of L-iduronate-2-sulfate sulfatase. Carbohyd. Res. in press.

16. Ginsberg, L.C., and Di Ferrante, N.: Sensitive methods for the determination of ester sulfate in biological systems. Biochem. Med., 17:80 (1977).

17. Hall, C.W., Cantz, M., and Neufeld, E.F.: A B-glucuronidase deficiency mucopolysaccharidosis: studies in cultured fibroblasts. Arch. Blochem. Biophys., 155: 32 (1973). 
18. Hall, C.W., and Neufeld, E.F.: $\alpha$-I-iduronldase activity in cultured skin fibroblasts and amniotic fluid cells. Arch. Blochem. Biophys., 158: 817 (1973).

19. Horton, D., and Philips, K.D.: 2,5-Anhydro-D-mannitol. Methods Carbohyd. Chem., 7: 68 (1976).

20. Kresse, H., and Neufeld, E.F.: The Sanfilippo A corrective factor. Purlfication and mode of action. J. Biol. Chem., 247: 2164 (1972).

21. Krystal, G., and Graham, A.F.: A sensitive method for estimating the carbohydrate content of glycoproteins. Anal. Blochem., 70: 336 (1976).

22. Lagunoff, D., Pritzl, P., and Scott, C.R.: Urinary N-sulfate glycosaminoglycan excretion in children: normal and abnormal values. Proc. Soc Exp. Biol. Med., 126: 34 (1967).

23. Lagunoff, D., and Warren, G.: Determination of 2-deoxy-2-sulfoaminohexose content of mucopolysaccharides. Arch. Biochem. Biophys., 99: 396
(1962).

24. Linker, A., and Hovingh, P.: The enzymatic degradation of heparitin sulfate. Blochim. Biophys. Acta, 165: 89 (1968).

25. Lowry, O.H., Rosebrough, N.J., Farr, A.L., and Randall, O.J.: Protein measurement with the Folin phenol reagent. J. Blol. Chem., 193: 265 (1951).

26. Matalon, R., Arbogast, B., Just1ce, P., Brandt, I.K., and Dorfman, A.:

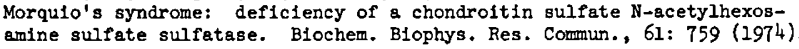

27. Matalon,-R., and Dorfman, A.: Hurler's syndrome, an $\alpha$-I-1duronidase deficiency. Biochem. Blophys. Res. Commun., 47: 959 (1972).

28. McKusick, V.A.: Heritable Disorders of Connective Tissue. C.v. Mosby Co., St. Louis, 4th Edition, pp. 521-686 (1972).

29. O'Brien, J.S.: Sanfilippo syndrome: profound deficiency of alpho-acetylglucosaminidase activity in organs and skin fibroblasts from type patients. Proc. Nat1. Acad. Sc1. USA, 69: 1720 (1972).

30. Pedrin1, V., Lenzi, L., and Zambott1, V.: Isolation and identification of keratosulfate in urine of patients affected by Morquio-Ullrich disease. Proc. Soc. Exp. B101. Med., 110: 847 (1962).

31. Pedrini-Mille, A., Pedrini, V., and Ponseti, I.V.: Glycosaminoglycans of lliac crest cartilage 1n normal children and in Morquio disease. J. Lab. Clin. Med., 84: 465 (1974).

32. Scott, J.A., Jr., and Melvin, E.H.: Determination of dextran with anthrone. Anal. Chem., 25: 1656 (1953).

33. Singh, J., Di Ferrante, N., Niebes, P., and Tavella, D.: N-acetylgalactosamine-6-sulfate sulfatase in man. Absence of the enzyme in Morqu10 disease. J. Clin. Invest., 57: 1036 (1976).

34. Singh, J., Donnelly, P.V., D1 Ferrante, N., N1chols, B.L., and Niebes, P.: Sanfillppo disesse: differentiation of types $A$ and $B$ by an analytical method. J. Lab. Clin. Med., 84: 438 (1974).

35. Singh, J., Tavella, D., and Di Ferrante, N.: Measurements of arylsulfatase $A$ and $B$ in human serum. J. Pediat., 86: 574 (1975).

36. Sjuberg, I., Fransson, L.-\&., Matalon, R., and Dorfman, A.: Hunter syndrome: a deficiency of I-1durono-sulfate sulfatase. Biochem. Biophys. Res. Commun., 54: 1125 (1973).

37. Stumpf, D.A., Austin, J.H., Crocker, A.C., and La France, M.: Mucopolysaccharidosis type VI (Maroteaux-Lamy syndrome). I. Sulfatase B def1clency in t1ssues. Am. J. D1s. Ch11d., 126: 747 (1973).

38. Suzuki, S., and Strominger, J.L.: Enzymatic sulfation of mucopolysaccharides in hen oviduct. J. B10l. Chem., 235: 267 (1960).

39. Trevelyan, W.E., Procter, D.P., and Harrison, J.S.: Detection of sugars on paper chromatography. Nature, 166: 444 (1950).

40. Wlesmann, U., and Neufeld, E.F.: Schele and Hurler syndromes: apparent 1dent1ty of the biochemical defect. Science, 169: 72 (1970).

41. Wolfe, L.S., Callahan, J., Fawcett, J.S., Andermann, F., and Scriver, C.R.: $G_{1}$ gangliosidosis without chondrodystrophy or visceromegaly. Neurology, 20: 23 (1970).

42. K\&K Laboratories, Inc., Plainview, N.Y. 11803.

43. St. Lou1s, Mo. 63178.

44. Fisher Scientific Co., Fair Lawn, N.J. 07410.

45. Plainview, N.Y. 11803.

46. New York, N.Y. 10017.

47. Bio-Rad Laboratories, Rockville Centre, N.Y. 11570.

48. Elkhart, Ind. 46514.

49. Boston, Mass. 02118.

50. Calbiochem, La Jolla, Ca. 92037.

51. We are grateful to $\mathrm{Dr}$. Arthur L. Beaudet for the assay of several lysosomal hydrolases.

52. This work was supported by U.S. National Institutes of Health grants GM-00081-03, HL-18692-01, HL-20447-01 and the Howard Hughes Med1cal Institute.

53. Requests for reprints should be addressed to: Dr. Nicola Di Ferrante, Department of Biochemistry, Baylor College of Medicine, Houston, TX. 77030 .

54. Received for publication November 16, 1977.

55. Accepted for publication March 21, 1978.
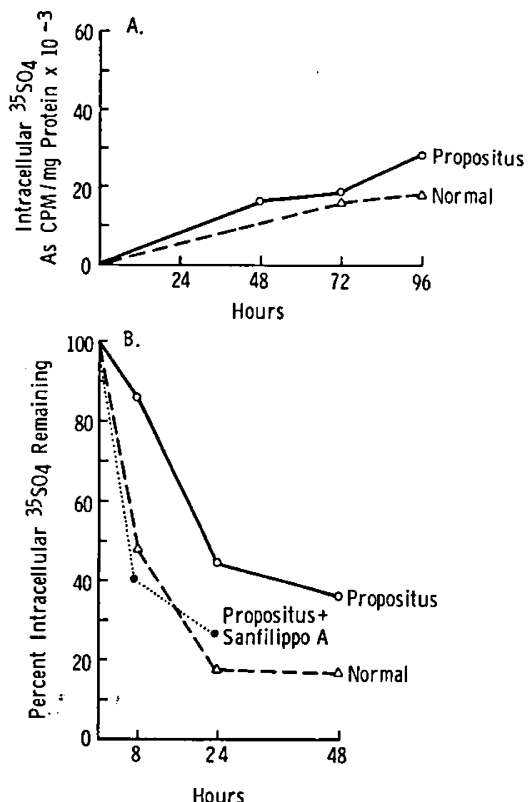

Fig. 1. Kinetics of $35 \mathrm{SO}_{4}$ uptake and release by the propositus' cultured fibroblasts. The uptake of $3 \mathrm{SO}_{4}$ is essentially normal (A), while the degrada-

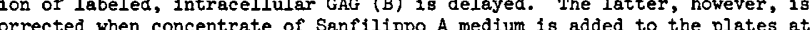
0 time.

HS

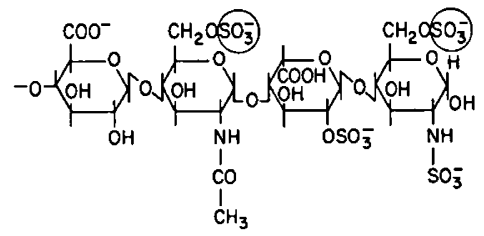

KS
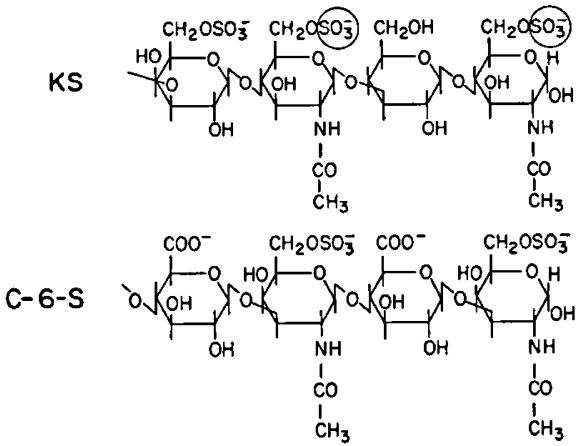

Fig. 2. The repeating units of heparan sulfate (HS), keratan sulfate (KS) and chondroftin-6-sulfate (C-6-S). The sulfate groups removed by N-acetylglucosamine-6-sulfate sulfatase are circled, while those removed by galactose6-sulfate or N-acetylgalactosamine-6-sulfate sulfatase are shadowed. The former ones are not cleaved in the disease described here; the latter ones are not cleaved in Morquio disease.

TABLE I

Polymeric GAG and ol1gosaccharides excreted by the propositus, as ms/24 hours.

\begin{tabular}{|c|c|c|c|}
\hline & \multicolumn{3}{|c|}{ GAG-containing: } \\
\hline & Hexuronate & 2-deoxy-2-sulfoamino hexose & galactose \\
\hline Polymeric (P) & 24.5 & 6.7 & $12.8^{2}+10.2^{2}$ \\
\hline Olfgosaccharldes $(0)$ & 23.2 & 1.4 & 7.0 \\
\hline Rat1o $\mathrm{P} / \mathrm{O}$ & 1.06 & 4.78 & 3.28 \\
\hline
\end{tabular}

1 CPC precipitable

2 Non-precipitable with CPC. 
TABLE II

Measurements of lysosomal enzymes on propositug'

serum or leukocytes extrects.

Results are expressed as nmoles substrate changed/hour/mg protein.

\begin{tabular}{|c|c|c|}
\hline Enzyrae & Propositus & Normal range \\
\hline a-L-iduronidase ${ }^{1}$ & 19.00 & $8-19.00$ \\
\hline I-1durono-2-sulfate sulfatase ${ }^{2}$ & 1.00 & $.80-.90$ \\
\hline a-N1-acetylglucosaminidase ${ }^{1}$ & 1950 & $462-2200$ \\
\hline B-galactosidase $\mathrm{e}^{2}$ & 400 & 250 \\
\hline $\begin{array}{l}\text { N-acetylgalactosamine-4-sulfate } \\
\text { sulfatase }{ }^{1} \text { (arylsulfatase B) }\end{array}$ & 100 & $70-160$ \\
\hline B-glucuronidase ${ }^{2}$ & 230 & $35-300$ \\
\hline
\end{tabular}

Assays performed on leukocytes extracts ${ }^{1}$ or serum ${ }^{2}$.
TABLE IV

Salient clinical and radiological features of Sanfilippo, Morquio and N-acetylglucosanine-6-gusfate sulfatase deficiency diseases.

\begin{tabular}{|c|c|c|c|}
\hline Clinical features & Santillppo & Morquio & $\begin{array}{l}\text { N-acetylglucosamine-6-sulfate } \\
\text { sulfatase deficiency }\end{array}$ \\
\hline Helght & normal & $<3$ rd percentile & - 3rd percentile \\
\hline Dysostosis multiplex & minimal & severe & mild \\
\hline Odontold process & normal & aplastic or hypoplastic & hypoplastic and dysplastic \\
\hline Mental retardation & severe & absent & present (I.Q. 50-60) \\
\hline Heperactivity & present & absent & present \\
\hline Hair & coarse, excessive & normal & coarse, excessive \\
\hline Cornea & clear & cloudy & clear \\
\hline Deafness & present & present & present \\
\hline Facies & normal to coarse & coarse & normal \\
\hline Hepatosplenomegaly & moderate & moderate & moderate \\
\hline Selzures & present & sosent & present \\
\hline
\end{tabular}

TABLE III

Measurements of galactose-6-sulfate, N-acetylgalactosamine-6-sulfate and N-acetylglucosamine-6-sulfate sulfatase on extracts of normal fibroblasts, Morquio flbroblasts, the propositus' fibroblasts and on extracts of his parents' leukocytes and fibroblasts. Results are expressed as nmoles substrate desulfated/hour/mg protein.

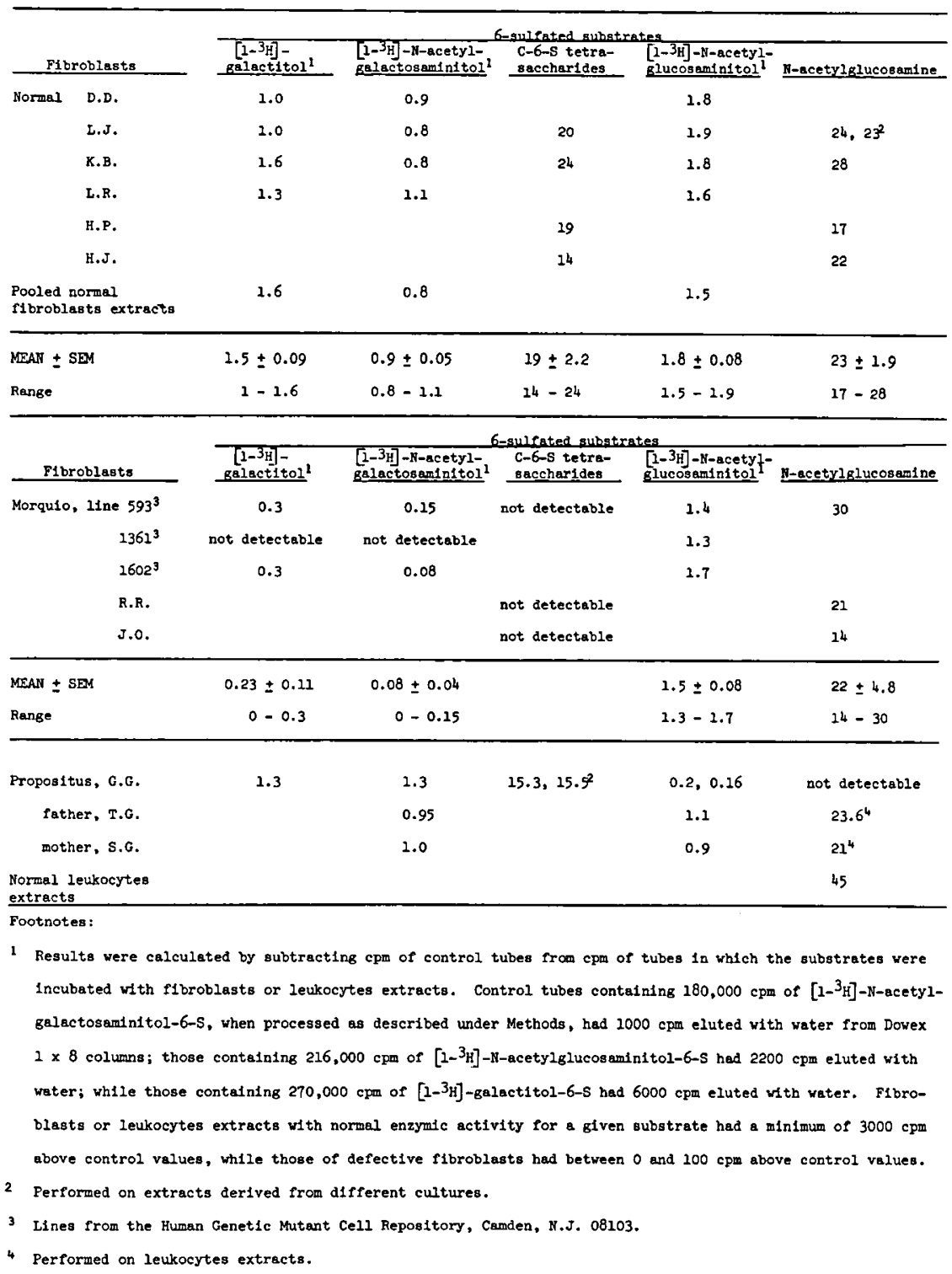

$031-3998 / 78 / 1207-0805 \$ 02.00 / 0$

:opyright (1) 1978 International Pediatric Research Foundation, Inc.

Printed in U.S.A. 